\title{
PKA-mediated phosphorylation of SPG11/spatacsin regulates binding with a subset of 14-3-3 proteins
}

Susanna Cogo ${ }^{1}$, James E. Tomkins ${ }^{2}$, Nikoleta Vavouraki ${ }^{2}$, Federica Forcellato ${ }^{1}$, Cinzia Franchin ${ }^{3}$, Isabella Tessari ${ }^{1}$, Giorgio Arrigoni ${ }^{3}$, Laura Cendron ${ }^{1}$, Claudia Manzoni ${ }^{4}$, Laura Civiero ${ }^{1,5}$, Patrick A. Lewis $^{2,6^{*}}$ \& Elisa Greggio ${ }^{1 *}$

${ }^{1}$ Department of Biology, University of Padova, Padova, Italy

${ }^{2}$ School of Pharmacy, University of Reading, Reading, United Kingdom

${ }^{3}$ Department of Biomedical Sciences, University of Padova, Padova, Italy

${ }^{4}$ School of Pharmacy, University College London, London, United Kingdom

${ }^{5}$ IRCCS San Camillo Hospital, Venice, Italy

${ }^{6}$ Department of Comparative Biomedical Sciences, Royal Veterinary College, London, United Kingdom

*Correspondence to: elisa.greggio@unipd.it; plewis@rvc.ac.uk

\begin{abstract}
$\underline{\text { Abstract }}$
Mutations in SPG11 constitute the major cause of autosomal recessive Hereditary Spastic Paraplegia (HSP) with thinning of the corpus callosum. Previous studies showed that SPG11/spatacsin orchestrates cellular traffic events through the formation of a coat-like complex and its loss of function results in lysosomal impairments. However, the upstream mechanisms that regulate SPG11/spatacsin trafficking are unknown. Here, using proteomics and CRISPR/Cas9-mediated tagging of endogenous SPG11/spatacsin, we identified a subset of 14-3-3 proteins as physiological interactors of SPG11. The interaction is modulated by PKA-dependent phosphorylation of SPG11 at Ser1955, which initiates SPG11 trafficking from the plasma membrane. Our study provides novel insight in understanding SPG11 physio-pathological roles with mechanistic dissection of its associated pathways.
\end{abstract}




\section{Introduction}

Loss of function mutations in SPG11, encoding SPG11/spatacsin, constitute the major cause of autosomal recessive Hereditary Spastic Paraplegia with thinning of the corpus callosum (ARHSPTCC). Although being largely heterogeneous, all HSPs are unified by the degeneration of distal axons in the corticospinal tracts, clinically resulting in slowly progressive spasticity primarily affecting the lower limbs (Blackstone, 2020; Stevanin et al., 2008). Genetically, >80 causative loci and over 60 genes have been identified and Mendelian modes of inheritance together with mitochondrial mutations have been described to date (Blackstone, 2018). Despite the large amount of genetic contributors, HSP genes have been associated with a relatively small number of cellular functions related to organelle biogenesis and membrane trafficking, lipid and mitochondria metabolism, development and myelination, as well as axonal transport and autophagy (Darios et al., 2020; Blackstone, 2018; Lo Giudice et al., 2014). SPG11/spatacsin is a 270kDa protein, highly expressed in the central nervous system, particularly in the motor cortex, spinal cord, hippocampus, cerebellum, dentate nucleus and pons (Murmu et al., 2011). Despite its association with multiple neurodegenerative disorders, including HSP, juvenile Parkinson's disease, Charcot-Marie-Tooth (CMT), amyotrophic lateral sclerosis (ALS) and Kjellin's syndrome (Patto \& O'kane, 2020), very little is yet known about the physiological role(s) of SPG11 and the consequences of its loss of function. Previous studies suggested an involvement of SPG11 in cellular traffic events, through the formation of a coat-like complex in association with two other HSP-linked proteins: SPG15/spastizin and the Adaptor Protein 5 (AP5) complex subunit zeta-1/SPG48 (Hirst et al., 2013). Interestingly, SPG11 and SPG15 patients show overlapping symptoms, whereas individuals affected by SPG48 forms typically display a later onset (Pensato et al., 2014; for a review on HSPs see Klebe et al., 2015). Accordingly, patient-derived fibroblasts as well as knockout (KO) animal models for SPG11 and SPG15 or their homologs display overlapping cellular phenotypes, with abnormally enlarged LAMP1-positive structures and/or the deposition of large intraneuronal membrane-surrounded materials that accumulate before the neuronal loss and are positive for LAMP1 (Khundadze et al., 2019, 2013; Renvoisé et al., 2014; Varga et al., 2015). Recently, Darios and collaborators generated a Spg $11^{-/}$mouse model that fully recapitulates the symptoms of HSP, linking lysosomal dysfunction to an impairment in lipid, and specifically ganglioside, clearance (Boutry et al., 2018; Branchu et al., 2017). Of interest, defects in both SPG11 and SPG15 have been associated with an impairment of the autophagic-lysosome reformation (ALR) process with the inhibition of tubule formation from late endosomes/lysosomes, a mechanism that is suggested to be AP5-independent (Boutry et al., 2019; Vantaggiato et al., 2019; Chang et al., 2014). Notably, the impairment in lysosome tubulation observed in absence of SPG11 has been associated with deficits in the clearance of lysosomal 
cholesterol and consequent alterations in its subcellular distribution (Branchu et al., 2017). In addition, the loss of SPG11 has been linked with axonal pathologies and reduced neurite complexity (Pérez-Brangulí et al., 2014), possibly dependent on a deregulation of the GSK3 $\beta / \beta$-catenin signalling pathway (Pozner et al., 2018). Nevertheless, currently available data on the subcellular localisation of SPG11 are conflicting. If, on one hand, this can be explained by the lack of sensitive tools for SPG11 detection and the so far poorly characterised SPG11 interactome, on the other hand it suggests a highly dynamic subcompartmentalisation. Thanks to the recently developed BioPlex 3.0, proteomescale, cell-line specific interaction network, the list of SPG11 candidate binding partners has greatly expanded, boosting the opportunities to further explore SPG11-related cellular pathways (Huttlin et al., 2020). Here, we adopted an analogous approach and performed a high-throughput affinity purification (AP) coupled to mass-spectrometry (MS) analysis to identify novel interactors for SPG11, which we assessed alongside literature-derived data obtained from the powerful Protein Interaction Network Online Tool (PINOT - (Tomkins et al., 2020)) and the BioPlex 3.0 dataset. After prioritisation of the candidate hits, we functionally validated the interaction between SPG11 and a subset of 14-3-3 chaperones. 14-3-3s are molecular shuttles which regulate localisation, activity and stability of their binding partners by interacting with specific phospho-motifs (Sluchanko \& Gusev, 2017). 14-3-3s are highly expressed in the brain during development and have been linked to multiple neurodegenerative processes (Civiero et al., 2017; Cornell \& Toyo-oka, 2017; Kaplan et al., 2017; McFerrin et al., 2017). We identified a 14-3-3 binding site in SPG11 and demonstrated that the interaction is modulated through phosphorylation of SPG11 at Ser1955 by cAMP-dependent protein kinase (PKA). Supporting in silico evidence predicting SPG11 as a transmembrane protein, we observed that PKA activation rapidly induces SPG11 translocation from the plasma membrane to the intracellular space. Our study provides novel insight into a developing understanding of SPG11 physio-pathological roles with mechanistic dissection of SPG11 associated pathways.

\section{$\underline{\text { Results }}$}

\section{AP-MS screening of SPG11 interactome nominates 14-3-3 proteins as candidate binding partners}

To obtain an unbiased overview of potential SPG11 related functional pathways, we interrogated its interactome, by performing a high-throughput screening of candidate protein-protein interaction (PPI) partners via affinity purification (AP) coupled with tandem mass spectrometry (MS/MS). After immunopurifying 3xFlag-tagged SPG11 or GFP as a negative control, we performed a pull-down assay by incubating the bait proteins with a murine brain lysate. Afterwards, we submitted the proteins bound to either SPG11 or GFP to MS/MS analysis. 84 proteins were considered positive hits in terms 
of potential SPG11 binding partners (see Materials and Methods for processing of MS/MS data). These interactors were converted to their human orthologs using the DSRC Integrative Ortholog Prediction Tool (DIOPT - Hu et al., 2011), whereby direct and high confidence mouse-human orthologs were identified in $97.6 \%$ of cases; 2 orthologs were assigned with moderate confidence (Supplementary File 1). Next, these proteins were used to query the CRAPome repository, a dataset of common MS contaminants (Mellacheruvu et al., 2013), which resulted in discarding 7 proteins from further analyses due to their prevalence as known contaminants in MS experiments (Supplementary File 2). The retained interactors were visualised in a PPI network, together with the extent of curated PPI literature for human SPG11, obtained from PINOT (6 interactors), and BioPlex 3.0 data (a further 38 interactors) (Figure 1A). Data from PINOT and BioPlex 3.0 were referred to collectively as literature-derived PPI data. The obtained list of SPG11 interactors (SPG11 interactome) was further analysed for subcellular localisation, based on Gene Ontology cellular components (GO CC) classification. GO CCs were grouped into semantic classes, based on semantic similarity, which were then grouped into location blocks (Supplementary File 3). We further investigated the semantic composition of the GO CC terms associated with SPG11 interactome by grouping terms related to neuronal structures or lysosomes into two additional location groups named "Neuron-related terms" and "Lysosomes". This analysis showed that the localisation of SPG11 has been associated with membranes, cytosol, nucleus, other organelles, vesicles, and cellular projections, and that SPG11 had GO CC terms related to neuronal structures and lysosomes (Figure 1B). Of note, 35 out of 77 of SPG11 interactors were associated with neuron related GO CC terms, 4 of which were 14-3-3 proteins $(4 / 35,11.4 \%)$.

\section{SPG11 interacts with a subset of 14-3-3s}

Based on MS screening and functional prioritisation of SPG11 candidate binding partners, we decided to pursue validation of the interaction between SPG11 and the 14-3-3 protein family. 14-3-3s are a group of seven adaptors that participate in multiple cellular functions, assisting the localisation, activity and/or stability of their binding partners (Civiero et al., 2017; Sluchanko \& Gusev, 2017). Relevant to SPG11 biology, 14-3-3s are highly expressed in the brain and are well-known to regulate the subcellular localisation of their client proteins through interaction with phosphorylated motifs (Sluchanko \& Gusev, 2017; Foote \& Zhou, 2012). Interestingly, six out of the seven isoforms of 143-3s were pulled down during AP-MS experiment (Figure 1A). We initially confirmed the interaction between 3xFlag-tagged SPG11 overexpressed in HEK293T cells and endogenous 14-3-3s, which were detected through a pan antibody designed against a common epitope shared by all the isoforms (Figure 2A). When assessing the subcellular localisation of 3xFlag-SPG11 in basal conditions, we found the protein to follow a punctate pattern with partial co-localisation with the endoplasmic 
reticulum (ER) and with sporadic enrichment at the cell periphery in protrusions-like structures (Figure 2B), in agreement with the GO enrichment of SPG11 interactors at cellular projections (Figure 1B). Of note, SPG11 staining partially colocalises with 14-3-3s, suggesting that under unstimulated conditions, only a pool of 14-3-3s interacts with SPG11 (Figure 2C). At this point, in order to determine which isoforms bind SPG11 with higher affinity, we purified via immobilisedmetal affinity chromatography (IMAC) all the seven 6xHis-tagged 14-3-3 family members and incubated them with affinity-purified 3xFlag-SPG11. We observed that SPG11 can bind all 14-3-3 isoforms, with the only exception of $\sigma-14-3-3$, confirming the MS results, and preferentially binds isoforms $\eta$ and $\gamma$, suggestive of a certain degree of specificity (Figure 2D).

\section{Phosphorylation at Ser1955 is key for SPG11 binding to 14-3-3s}

Considering that 14-3-3s typically interact with their partners at specific phospho-sites, commonly phospho-serines, through recognition of conserved consensus sequences (Civiero et al., 2017; Sluchanko \& Gusev, 2017), we interrogated the 14-3-3-Pred tool for the prediction of candidate 143-3 binding sites in SPG11 (http://www.compbio.dundee.ac.uk/1433pred - Madeira et al., 2015) and the PhosphoSitePlus ${ }^{\circledR}$ database, which curates information on protein post-translational modifications, including phosphorylation. In parallel, we used phospho-peptide enrichment coupled with liquid-chromatography (LC)-MS/MS analysis on purified 3xFlag-SPG11, to identify putative phosphorylated peptides. Figure 3A summarises the results obtained by the combination of in silico and experimental data. Among the seven candidate 14-3-3 binding sites predicted by 14-3-3-Pred, only Ser1955 (highlighted in light blue) (i) was previously reported to be a phosphorylated residue in cells and (ii) was identified by our phospho-peptide enrichment. To validate S1955 as a binding site for 14-3-3s, we generated the phospho-deficient S1955A (SA) mutant by site direct mutagenesis. We next performed pull-down assays between recombinant 3xFlag-SPG11 and 6xHis-14-3-3 isoforms to compare the binding affinity of WT versus mutant SPG11 for 14-3-3s. Our results indicate that loss of phosphorylation on Ser1955 decreases 14-3-3 binding to SPG11 by 50\% (Figure 3B).

\section{PKA phosphorylates SPG11 at Ser1955}

So far, the upstream mechanisms orchestrating SPG11 trafficking and compartmentalisation are poorly characterised. Based on the conserved mechanism through which 14-3-3s bind SPG11 via a phospho-motif, we employed the NetPhos 3.1 Server (Blom et al., 2004, 1999) to seek for the putative upstream kinase(s) responsible for phosphorylation of SPG11 at Ser1955. The results of the prediction, depicted in Figure 4A, suggested PKA to be the upstream kinase phosphorylating SPG11 at this residue with the highest confidence score. At this point, we adopted a combination of cell biology and pharmacological approaches to validate the signalling cascade. Due to the lack of sensitive and selective antibodies against SPG11, we generated monoclonal CRISPR/Cas9 genome- 
edited HeLa cell lines by inserting a 3xFlag-2xStrep tag at the N-terminus of the SPG11 sequence. To increase the likelihood of correct genome-editing, we designed two independent guide RNAs for the targeting of Cas9 in the proximity of the SPG11 ATG start codon. A schematic of the experimental approach, together with the validation of CRISPR/Cas9-edited lines, are shown in Supplementary Figure 1A-C. As predicted by the design platform, sgRNA2 turned to be more efficient, and clone 3 was selected for the following experiments (Supplementary Figure 1C). We subsequently immunopurified endogenous SPG11 from this cell line, taking advantage of the 3xFlag tag, in absence or presence of PKA pharmacological activation achieved with a combination of Forskolin and Isobutylmethylxanthine (IBMX), and monitored the binding of recombinant $\eta-14-3-3$ to SPG11. Our data revealed that PKA activation clearly increases $\eta-14-3-3$ binding to SPG11 (Figure 4B), confirming the prediction that PKA phosphorylates SPG11 on Ser1955. In parallel, we took advantage of the CRISPR/Cas9-tagged SPG11 lines to evaluate the interaction between endogenous SPG11 and endogenous 14-3-3s in absence or presence of PKA activation by means of proximity ligation assay (PLA). As reported in Figure 4C, not only we could confirm the formation of the SPG11/14-3-3 complex at the endogenous level, but we could also strengthen the evidence that PKA activity increases the affinity of SPG11/14-3-3 complex.

\section{PKA-mediated phosphorylation initiates SPG11 traffic from the plasma membrane}

We next wanted to evaluate the functional consequence of PKA phosphorylation on SPG11. PKA, the main cellular effector of cyclic AMP (cAMP), orchestrates several signalling pathways through phosphorylation of multiple substrates (Patra \& Brady, 2018; Sassone-Corsi, 2012). In neurons, PKA produces a range of fast and dynamic responses at the plasma membrane, cytosol and the nucleus (seconds at membrane level, few minutes at cytosol and nucleus level), according to a tight spatiotemporal regulation of cAMP levels (Gervasi et al., 2007). In contrast, immortalised cell lines respond to PKA activation with slightly longer timescales (Hamaguchi et al., 2015; Namkoong et al., 2009). Previous evidence from bioinformatic studies suggests that SPG11 is a transmembrane protein (Stevanin et al., 2007). Using the PSIPRED and the Phobius software (http://bioinf.cs.ucl.ac.uk/psipred/ - Buchan \& Jones, 2019; http://phobius.sbc.su.se/cgibin/predict.pl), we confirmed that SPG11 is predicted to be a transmembrane protein with at least two confirmed transmembrane domains. Because a proportion of SPG11 appears to cluster in cellular protrusions consistent with a localisation at the plasma membrane (Figure 2B), we hypothesised that PKA-mediated phosphorylation on SPG11-Ser1955 could act as a signal for SPG11 relocalisation. To this aim, we employed cell-surface biotinylation assay to measure (i) membrane versus intracellular SPG11 fractions and (ii) the response to PKA activation over time, via monitoring the internalisation of 3xFlag-2xStrep-tagged endogenous SPG11 after 5, 15 and 90 minutes of 
Forskolin/IBMX treatment. Figure 5A shows that (i) SPG11 is recovered in the membrane fraction and (ii) PKA activation reduces the plasma membrane fraction of SPG11 within 15 minutes, suggesting its internalisation. In parallel, to confirm that this process is mediated by a 14-3-3dependent mechanism, we imaged the localisation of SPG11 in HeLa cells transfected with WT- or SPG11-S1955A, treated over time with Forskolin/IBMX. In agreement, the amount of SPG11 located at the cell protrusions is reduced after 15 minutes of PKA activation and starts to recover 90 minutes after stimulation. Importantly, localisation of SPG11-S1955A is unchanged upon Forskolin/IBMX treatment supporting a mechanism where PKA-mediated phosphorylation of Ser1955 is the signal that induces SPG11 trafficking from the plasma membrane to the intracellular compartments (Figure 5B).

\section{$\underline{\text { Discussion }}$}

Loss of function mutations in SPG11 represent the most common cause of ARHSP-TCC, and have been linked to multiple neurodegenerative disorders (Branchu et al., 2017). Previous studies demonstrated that lack of SPG11 leads to deficits in vesicle trafficking, ALR and lipid clearance (Boutry et al., 2019, 2018; Chang et al., 2014; Hirst et al., 2013). However, detailed indications on SPG11 compartmentalisation and the mechanism of its regulation and recruitment are currently unavailable. We employed an unbiased, AP-MS approach and probed SPG11 as a bait against a whole brain sample, with the aim to identify binding partners of SPG11 in basal conditions. We then combined experimental data with cutting-edge bioinformatics approaches to guide the selection of candidate interactors to be validated in a cellular system. We identified a list of 77 putative novel interactors, which we prioritised according to their subcellular localisation. Intriguingly, we found the SPG11 interactome to be highly diverse in terms of subcellular compartmentalisation. SPG11 itself was associated with multiple organelles, suggestive of a dynamic shuttling across compartments. Among all groups, we prioritised the hits that are most likely relevant in a neuronal context, based on SPG11's robust link to neurodegeneration, and narrowed down the list to 35 proteins. Strikingly, members of the family of 14-3-3 adaptor proteins represented the $11.4 \%$ of proteins with neuron-related GO CC terms. 14-3-3s are abundantly expressed in the brain, where they cover fundamental roles during development, and they have been implicated in a variety of neurological disorders, based on genetic evidence, their relationship with relevant disease proteins and/or pathological findings (Foote \& Zhou, 2012). Using phospho-peptide enrichment analysis and biochemical approaches, we identified and validated a phospho-residue at the C-terminus of SPG11, namely Ser1955, as the main 14-3-3 binding site. Of note, depletion of Ser1955 phosphorylation, by means of Ser-to-Ala phospho-deficient mutant, significantly reduced, although not completely 
abolished, the interaction. This can be explained by the fact that 14-3-3s usually function as dimers each of them binding one phospho-peptide (Sluchanko \& Gusev, 2012). Thus, additional 14-3-3 binding motifs are likely to be present within SPG11 sequence, as also supported by in silico prediction from the 14-3-3-Pred tool (Figure 3A). Further supporting our results, an independent analysis performed with the Eukaryotic Linear Motifs (ELM - Kumar et al., 2020) software also suggests the presence of multiple 14-3-3 binding sites within SPG11, including the RVHSTSSL peptide containing Ser1955. We additionally proved Ser1955 to be phosphorylated by PKA. PKA activation is determined by a raise in cAMP levels, whereas specificity in the signalling is achieved through PKA-anchoring proteins (AKAPs), which place PKA in close proximity to effectors and substrates (Sassone-Corsi, 2012). Independent bioinformatic predictions performed by us and others suggest that SPG11 is a transmembrane protein, with multiple transmembrane domains (Figure 6). Based on the proposed roles of SPG11 on cellular trafficking, via AP5 and SPG15, on ALR, through binding with SPG15, and on our observation of a partial but consistent distribution of the protein at the level of the plasma membrane, we hypothesised that the interaction between SPG11 and 14-3-3s could play a pivotal role in the cellular localisation of SPG11, with PKA-mediated phosphorylation acting as the triggering signal. We therefore demonstrated that PKA activation is capable of mediating SPG11 internalisation, taking advantage of a powerful cell-surface biotinylation assay, and that this happens in a 14-3-3-dependent manner (Figure 5A). Interestingly, PKA activation was found to be protective against lipid-induced toxicity in a model of a lysosomal storage disorder, due to its ability of re-acidifying disrupted lysosomal pH (Folts et al., 2016). Lipid dyshomeostasis has recently been proposed to represent a common denominator in the physiopathology of HSP (Darios et al., 2020). Therefore, given the interplay between SPG11 and other spastic paraplegia genes, further mechanistic dissection of the PKA/SPG11/14-3-3 cascade could shed light on the broader pathways that are impaired during disease. Gaining greater insight into the upstream signals that regulate this pathway, and whether these are AP5-dependent or independent events, will support the development of novel approaches to target their dysfunction in disease.

\section{Experimental procedures}

\section{Animals}

Housing and handling of mice were done in compliance with national guidelines. Animal procedures were approved by the Ethical Committee of the University of Padova and the Italian Ministry of Health (license 1041216-PR). 


\section{Plasmids and reagents}

The sequence of human SPG11 was cloned into a pReceiver-M12 Expression Clone vector (GeneCopoeia). 3xFlag-GFP was used as a negative control in pull-down experiments. Generation of SPG11-S1955A mutant was outsourced to VectorBuilder. Cloning of 6xHis-tagged 14-3-3 isoforms in a pET28a(+) vector for bacterial expression was performed as described in (Civiero et al., 2017). For the generation of CRISPR/Cas9 edited lines, sgRNAs and the HDR template were synthesised by Sigma-Aldrich. sgRNAs were subsequently cloned into the pSpCas9(BB)-2A-Puro(PX459)V2.0 vector (Addgene). $2 \mu \mathrm{g} / \mathrm{ml}$ puromycin (InvivoGen) was employed for the selection of positive clones. Treatment of cells with Forskolin and IBMX (both purchased from Sigma-Aldrich) was performed for the times indicated in figure captions at 30 and $100 \mu \mathrm{M}$, respectively.

\section{Cell culture and transfection}

HeLa and HEK293T cells purchased from ATCC were cultured in Dulbecco's modified Eagle's medium (DMEM, Life Technologies) supplemented with $10 \%$ foetal bovine serum (FBS, Life Technologies). Cell lines were maintained at $37^{\circ} \mathrm{C}$ in a $5 \% \mathrm{CO}_{2}$ controlled atmosphere. $0.25 \%$ trypsin (Life Technologies), supplemented with $0.53 \mathrm{mM}$ EDTA, was employed to generate subcultures. Cells were transiently transfected with plasmid DNA using polyethylenimine (PEI, Polysciences), with a 1:3 DNA:PEI ratio. For pull-down assays, cells were plated onto $150 \mathrm{~mm}$ dishes and transfected

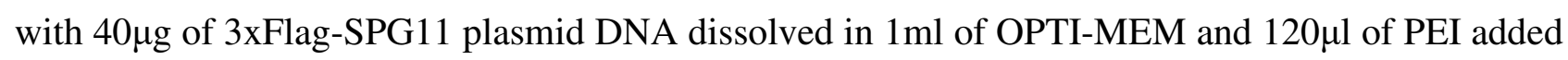
to $1 \mathrm{ml}$. In the case of the $3 \mathrm{xFlag}$-GFP control construct, which displays a higher efficiency in terms of both transfection and expression, only $10 \mu \mathrm{g}$ DNA were transfected, to obtain comparable purification yields. 48-72h after transfection, cells were harvested in RIPA buffer (Cell Signaling Technologies), supplemented with protease inhibitor cocktail (Sigma-Aldrich) and processed for the pull-down reactions.

\section{Generation of 3xFlag/2xStrep-SPG11 CRISPR/Cas9 edited monoclonal lines}

Insertion of a $3 \times$ Flag/2xStrep tag at the N-terminus of endogenous SPG11 was performed using CRISPR/Cas9-mediated genome editing technology through homology-directed repair (HDR), following the protocol by Sharma et al., 2018. sgRNAs were designed taking advantage of the online platform Benchling (www.benchling.com) and scored based on (Doench et al., 2016; Hsu et al., 2013). Two sgRNAs were selected to increase the probability of success. As predicted by Benchling, sgRNA2 was found to be more specific and efficient in targeting SPG11 (Supplementary Figure 1A). The 192bp tag sequence to be used as HDR template was obtained from (Dalvai et al., 2015) and is flanked by two homology arms (HA), having a length of 600bp each and designed based on SPG11 genomic sequence. 


\section{Purification of recombinant 14-3-3s}

6xHis-14-3-3 isoforms were expressed in BL21(DE3) bacterial cells and purified in batch by IMAC with ProBond ${ }^{\mathrm{TM}}$ resin (Invitrogen). After elution with imidazole, buffer was exchanged to PBS with a PD10 desalting column (GE Healthcare). Proteins were quantified measuring UV absorption and subsequently supplemented with $3 \mathrm{mM}$ dithiothreitol (DTT); proteins were quickly frozen and kept at $-80^{\circ} \mathrm{C}$ for long-term storage. 1:20 molar ratio between SPG11 and 14-3-3s was employed for pulldown experiments.

\section{Cell lysis, protein purification and pull-down assays}

For the purification protocol, cell lysates were incubated on ice for 30 minutes and subsequently cleared by centrifugation at $20000 \mathrm{~g}$ for 30 minutes at $4^{\circ} \mathrm{C}$, after which the supernatants were incubated for 2 hours with $40 \mu$ l of anti-Flag® M2 Affinity Gel (Sigma-Aldrich) at $4^{\circ} \mathrm{C}$. After that, immunocomplexes were washed ten times in buffers with decreasing ionic strength and incubated with mouse brain lysates, or washed three times prior to proceeding to pull-downs with recombinant or endogenous 14-3-3s. The pull-down reaction was conducted overnight in a binding buffer containing 20mM Tris- $\mathrm{HCl}, \mathrm{pH} 7.5,150 \mathrm{mM} \mathrm{NaCl}, 1 \mathrm{mM}$ EDTA, 2.5mM sodium pyrophosphate, $1 \mathrm{mM} \beta$-glycerophosphate, $1 \mathrm{mM}$ sodium orthovanadate and $1 \% \mathrm{NP}-40$. The following morning, resins were washed three times with the binding buffer and processed for Western Blot analysis.

\section{Cell-surface biotinylation assay}

To evaluate the presence of SPG11 at the plasma membrane and its internalisation upon PKA activation, we performed the cell-surface biotinylation assay utilising the EZ-Link Sulfo-NHS-SSBiotin (ThermoFisher Scientific) as per manufactures' recommendations. Briefly, genome-edited cells were plated onto 6-well plates and subjected to treatment with PKA activators (Forskolin/IBMX) for $0,5,15$ and 90 minutes. Cells were then transferred on ice and washed three times with ice-cold PBS pH 8.2, supplemented with $1 \mathrm{mM} \mathrm{MgCl}$ and $0.1 \mathrm{mM} \mathrm{CaCl}_{2}\left(\mathrm{PBS}^{2+}\right)$. Sulfo-Biotin was added for 30 minutes on ice at a concentration of $0.3 \mathrm{mg} / \mathrm{ml}$ in $\mathrm{PBS}^{2+}$ in the dark. After three more washes in $\mathrm{PBS}^{2+}$, cells were harvested in RIPA buffer without EDTA, and biotinylated proteins were immunopurified through Streptavidin-Mag Sepharose ${ }^{\mathrm{TM}}$ (GE Healthcare). After washing of the beads, samples were processed for Western Blot. For each sample, input indicating the total amount of SPG11 and flow-through, representing the intracellular fraction of the protein, were also collected.

\section{Western Blot}

15 to $30 \mu \mathrm{g}$ of total protein samples were resolved on ExpressPlus ${ }^{\mathrm{TM}}$ PAGE 4-20\% gels (GenScript), in MOPS running buffer, or $8 \%$ Tris-glycine polyacrylamide gels in SDS/Tris-glycine running buffer, according to the size-resolution required. The resolved proteins were transferred to polyvinylidenedifluoride (PVDF) membranes (Bio-Rad), through a Trans-Blot ${ }^{\circledR}$ Turbo ${ }^{\mathrm{TM}}$ Transfer 
System (Bio-Rad). PVDF membranes were subsequently blocked in Tris-buffered saline plus $0.1 \%$ Tween (TBS-T) and 5\% non-fat milk for 1 hour at $4^{\circ} \mathrm{C}$ and then incubated over-night at $4{ }^{\circ} \mathrm{C}$ with primary antibodies in TBS-T plus 5\% non-fat milk. Membranes were then washed in TBS-T (3x10 minutes) at room temperature (RT) and subsequently incubated for 1 hour at RT with horseradish peroxidase (HRP)-conjugated $\alpha$-mouse or $\alpha$-rabbit IgG. Blots were then washed in TBS-T (4x10 min) at RT and rinsed in TBS-T, and immunoreactive proteins were visualised using Immobilon® Forte Western HRP Substrate (Merck Millipore). Densitometric analysis was carried out using the ImageJ software. The antibodies used for Western Blot are as follows: mouse $\alpha$ - $\beta$-actin (A1978, SigmaAldrich, 1:20000), mouse $\alpha$-pan 14-3-3 (sc-1657, Santa Cruz Biotechnology, 1:1000 to 1:10000), $\alpha$ Flag® M2-HRP (A8592, Sigma-Aldrich, 1:20000), mouse $\alpha$-His-HRP (A7058, Sigma-Aldrich, 1:10000).

\section{Protein digestion, phospho-peptide enrichment and LC-MS/MS analysis}

Gel bands were subjected to in-gel digestion with sequencing-grade modified trypsin (Promega) as detailed in Belluzzi et al., 2016. A similar procedure was used for the digestion of phosphorylated proteins, but in this case LysC was used as protease. Briefly, gel bands were cut in small pieces, dehydrated with acetonitrile (ACN), and dried under vacuum. Reduction of cysteines was performed with freshly prepared $10 \mathrm{mM}$ DTT in $25 \mathrm{mM}$ Tris- $\mathrm{HCl} \mathrm{pH} 8.5$, at $56^{\circ} \mathrm{C}$ and for $1 \mathrm{~h}$. Alkylation was performed with 55mM iodoacetamide (Sigma Aldrich) in $25 \mathrm{mM}$ Tris- $\mathrm{HCl} \mathrm{pH} 8.5$ for $45 \mathrm{mins}$ at room temperature and in the dark. Gel pieces were washed several times with $25 \mathrm{mM}$ Tris- $\mathrm{HCl} \mathrm{pH} 8.5$ and ACN, dried under vacuum, and suspended LysC solution (Promega, $12.5 \mathrm{ng} / \mathrm{mL}$ in $25 \mathrm{mM}$ Tris- $\mathrm{HCl}$ $\mathrm{pH}$ 8.5). Digestion was performed overnight at $37^{\circ} \mathrm{C}$. Peptides were extracted with three changes of $50 \% \mathrm{ACN} / 0.1 \%$ formic acid (FA, Fluka). Samples were dried under vacuum and stored at $-20^{\circ} \mathrm{C}$ till the phosphopeptide enrichment procedure was performed. Phosphopeptide enrichment and LCMS/MS analysis were performed as detailed in Belluzzi et al., 2016. MS analysis of the samples was performed with an LTQ-Orbitrap XL mass spectrometer (Thermo Fisher Scientific) coupled online with a nano-HPLC Ultimate 3000 (Dionex-Thermo Fisher Scientific). Raw data files were analysed with Proteome Discoverer software (version 1.4, Thermo Fisher Scientific) connected to a Mascot Server version 2.2.4 (Matrix Science, UK) against the Uniprot Mouse Database (version 2015.04.01). Trypsin or LysC were set as digesting enzymes with up to one missed-cleavage for protein identification and up to three missed-cleavages for phosphopeptides identification. Carbamidomethyl cysteine was set as fixed modification and methionine oxidation as variable modification in all cases. Phosphorylation of Ser/Thr/Tyr residues was set as variable modifications when the phosphopeptide analysis was performed. Peptide and fragment tolerance were $10 \mathrm{ppm}$ and $0.6 \mathrm{Da}$, respectively. The algorithm Percolator was used to calculate False Discovery Rate (FDR) and PhosphoRS algorithm 
(Taus et al., 2011) was used to help in the assignment of the correct phosphorylation sites. Proteins were considered as correctly identified if at least 2 unique peptides per protein were sequenced with a $q$ value $<0.01$. Quantification was achieved by integrating the area under the peaks for each identified peptide and then by averaging the values obtained for all peptides belonging to the same protein. A principle of maximum parsimony was applied to group proteins into protein families. The threshold for determining positive hits, in terms of SPG11 binding partners, was a minimum of 5 peptides matching the assigned protein and a minimum signal fold change above control of 5 .

\section{Protein-protein interaction (PPI) networks and functional prioritisation of the data}

Murine proteins identified by MS were converted to their respective human orthologs using the DSRC Integrative Ortholog Prediction Tool (DIOPT) version 8 (Hu et al., 2011); input species Mus musculus, output species Homo sapiens, with the option of all prediction tools selected to provide a broad meta-analysis. Ortholog assignments were determined based on the highest confidence predictions (Supplementary File 1). These orthologs were queried against the 411 datasets in the CRAPome repository, version 1.1 (Mellacheruvu et al., 2013) to identify likely contaminants in our MS analysis. Proteins present in $>50 \%$ of the CRAPome datasets were discarded from further analyses (Supplementary File 2). Retained proteins were considered alongside literature-derived data, obtained from the Protein Interaction Network Online Tool (PINOT - Tomkins et al., 2020) and BioPlex 3.0 dataset (Huttlin et al., 2020), for network visualisation. PINOT was queried on 22nd May 2020, with Homo sapiens and stringent filtering parameters selected. For the retained interactors of SPG11 from the AP/MS analysis, we collected the Gene Ontology cellular component (GO CC) terms through AmiGO v2 on $6^{\text {th }}$ August 2020, and for SPG11 on $21^{\text {st }}$ August 2020. The GO CC terms were grouped based on semantic similarity into semantic classes, which were further grouped into location blocks. Two additional location blocks were created, named "Neuron-related terms" and "Lysosome" in which we added any term that was related to neurons, or lysosomes, respectively. The existence of GO CC terms that belong in a location block was then qualitatively visualised in Cytoscape using pie charts inside each node. Network and location block visualisations were generated in Cytoscape v3.8.0 and proteins were defined using HGNC approved nomenclature.

\section{Bioinformatic predictions}

Evaluation of putative transmembrane domains in SPG11 was performed with the PSIPRED predictor of secondary structure (Buchan \& Jones, 2019) and Phobius, a combined transmembrane topology and signal peptide predictor developed by the Stockholm Bioinformatics Centre. Analysis of putative phosphorylations and the upstream kinases was conducted employing NetPhos 3.1 (ExPASy Bioinformatics Resource Portal). The Eukaryotic Linear Motif resource for Functional Sites in Proteins (ELM - Kumar et al., 2020; Puntervoll et al., 2003) and 14-3-3-Pred 
(http://www.compbio.dundee.ac.uk/1433pred - Madeira et al., 2015) were also interrogated for the prediction of 14-3-3 binding-motifs.

\section{Immunocytochemistry, PLA and confocal microscopy}

For immunocytochemistry (ICC), cells were cultured onto $12 \mathrm{~mm}$ glass coverslips in 12-well plates coated with poly-L-lysine (Sigma-Aldrich). Cells were fixed after $48 \mathrm{~h}$ of transfection using $4 \%$ paraformaldehyde (PFA) and subsequently subjected to staining. The antibodies and dyes used for ICC are as follows: mouse $\alpha$-pan 14-3-3 (sc-1657, Santa Cruz Biotechnology, 1:100), mouse $\alpha$-Flag® M2 (F1804, Sigma-Aldrich, 1:100), polyHis-HRP (A7058, Sigma-Aldrich, 1:10000), rabbit $\alpha$ DYKDDDDK tag - alias Flag - (14793S, CST, 1:100), Hoechst (33258, Invitrogen) and phalloidiniFluor 647 (ab176759, abcam). For PLA, CRISPR/Cas9-edited lines were subjected to staining with the Duolink ${ }^{\mathrm{TM}}$ In Situ Detection Reagents Orange (DUO92007) and the combination of $\alpha$-mouse and $\alpha$-rabbit probes (DUO92002 \& DUO92004) from Sigma-Aldrich, as per manufacturers' recommendations. Proteins and complexes were afterwards visualised by confocal microscopy (Zeiss LSM700). Quantification of PLA positive signal was performed with ImageJ software.

\section{Funding}

This work was supported by: Intramural Research Program, Department of Biology, University of Padova [PRID 2017] to EG, CARIPARO PhD Fellowship to SC; the Biomarkers Across Neurodegenerative Diseases Grant Program 2019, BAND3 (Michael J Fox Foundation, Alzheimer's Association, Alzheimer's Research UK and Weston Brain Institute [grant number 18063 to CM and PAL]); Biotechnology and Biological Sciences Research Council CASE studentship with BC Platforms to JET [grant number BB/M017222/1]; Engineering and Physical Sciences Research Council studentship to NV [grant number EP/M508123/1]; the Medical Research Council [grant number MR/N026004/1 to PAL]; and the Dolby Family Foundation [studentship to NV]. 
bioRxiv preprint doi: https://doi.org/10.1101/2020.09.09.289009; this version posted September 10,2020. The copyright holder for this preprint (which was not certified by peer review) is the author/funder, who has granted bioRxiv a license to display the preprint in perpetuity. It is made available under aCC-BY-ND 4.0 International license.
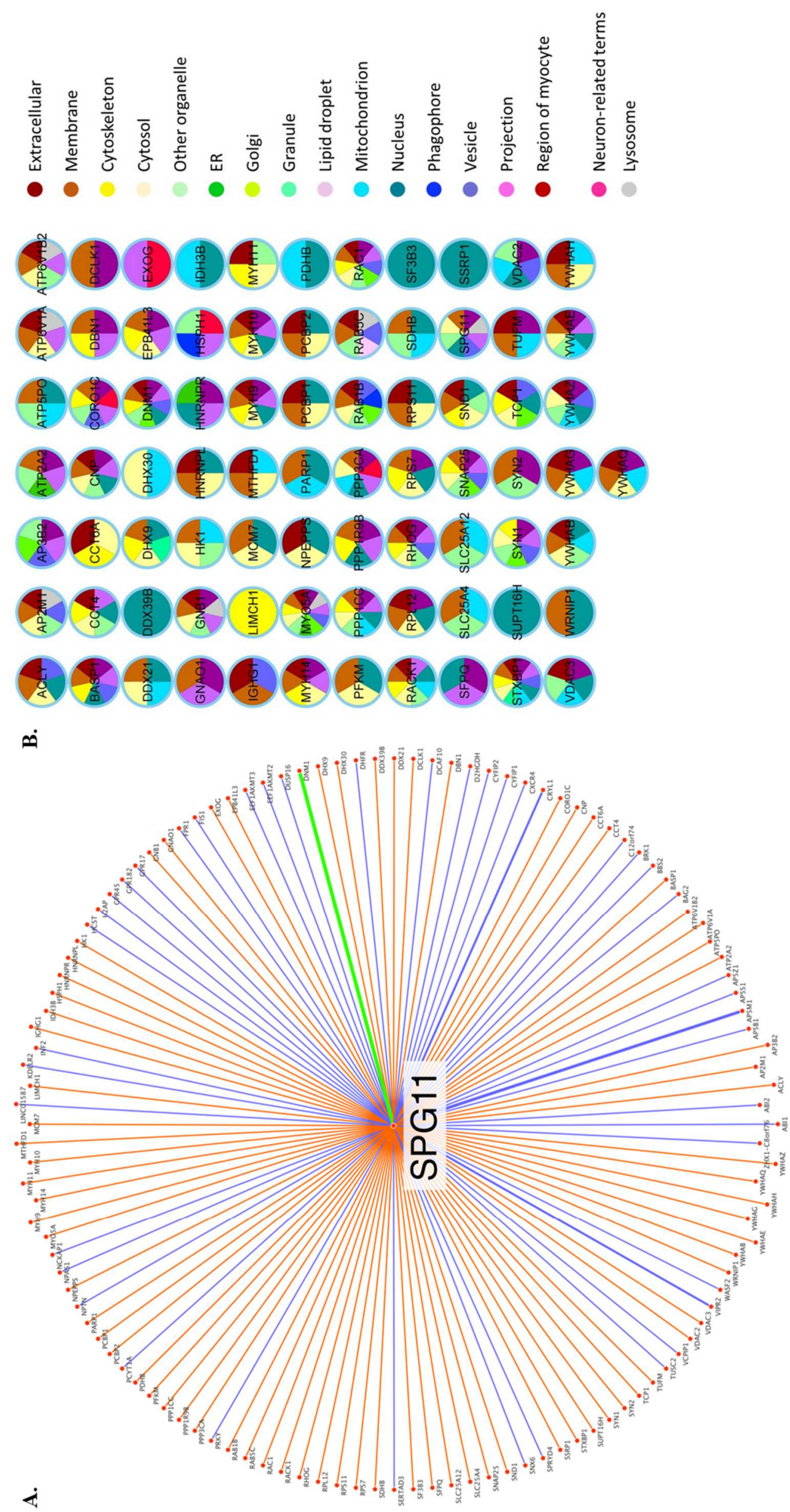
Figure 1 - The SPG11 interactome. (A) A protein interaction network of SPG11 illustrating novel protein interactors identified by mass spectrometry in this study (orange edges), literature-derived protein interactors from PINOT and BioPlex 3.0 (blue edges), and an interactor common to both our novel dataset and literaturederived data (green edge). The edge thickness positively correlates with protein interaction confidence based on distinct method detection strategies used and the number of specific documented reports. (B) The subcellular localisation of the interactors resulted from our AP/MS analysis and of SPG11 is visualised, with each colour corresponding to a different location block. The collection of subcellular location data in the form of Gene Ontology cellular component terms was performed through AmiGO and the grouping of each term into location blocks with an in-house grouping protocol. 


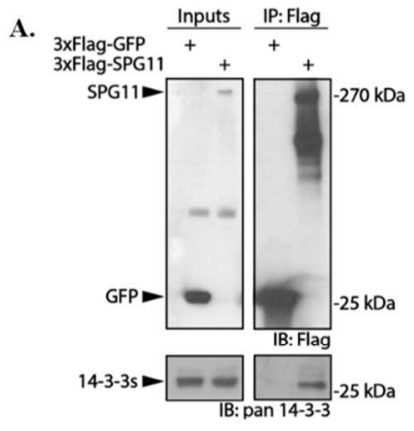

C.
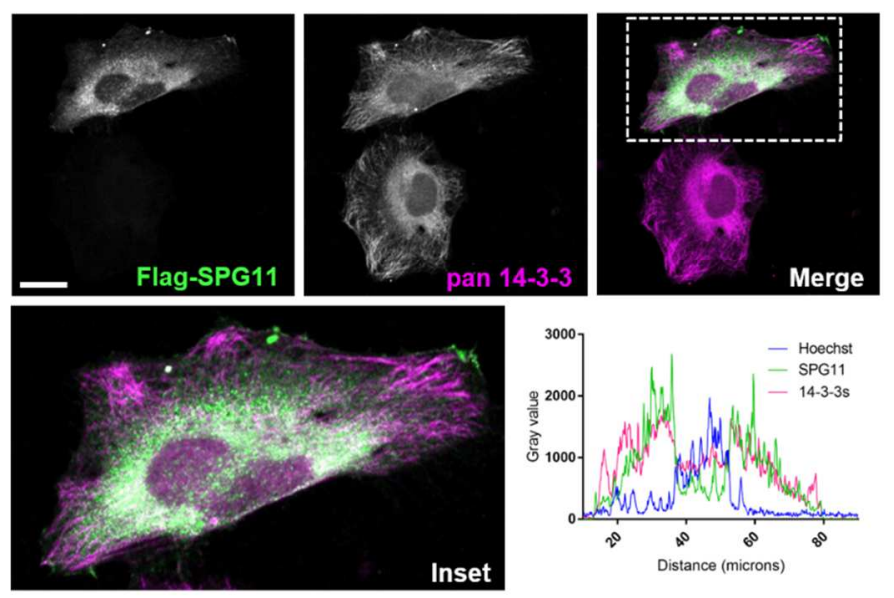
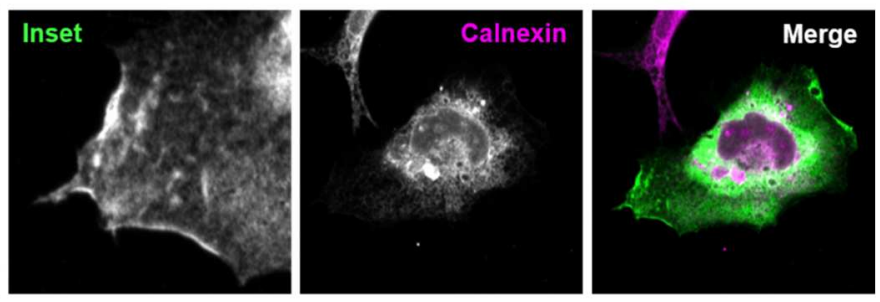

D.
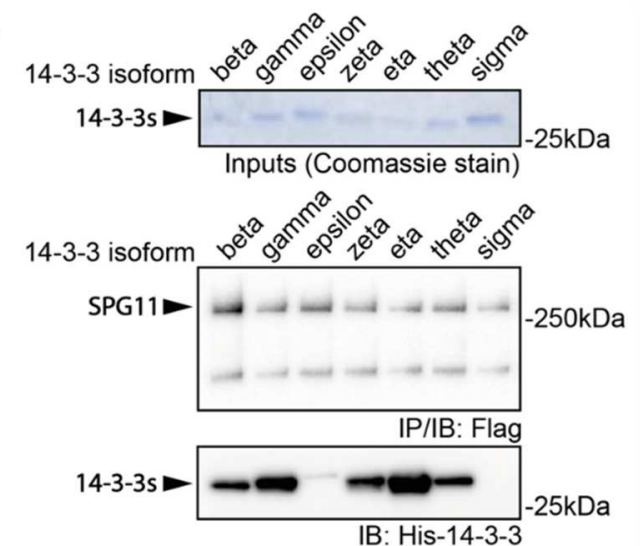

IB: His-14-3-3

Figure 2 - SPG11 interacts with a subset of 14-3-3s in cells. (A) 3xFlag-SPG11 overexpressed in HEK293T cells pulls down endogenous 14-3-3 proteins. (B) Immunocytochemical evaluation of 3xFlag-SPG11 subcellular compartmentalisation in HeLa cells reveals predominant perinuclear localisation, partially overlapping with the ER marker calnexin, and sporadic enrichment at the cell periphery in protrusions-like structures. (C) Co-staining of 3xFlag-SPG11 and 14-3-3s suggests that SPG11 only overlaps with a subset of 14-3-3s, as confirmed via pull-down assays between 3xFlag-SPG11 and recombinant 14-3-3 isoforms purified by IMAC (D). Scale bars $=20 \mu \mathrm{m}$. 
A.

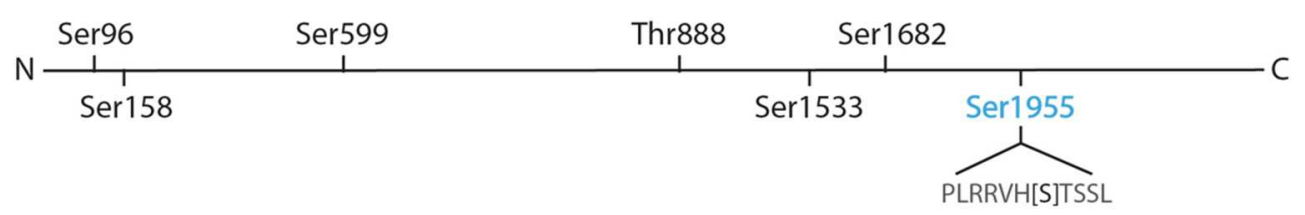

B. 14-3-3 isoform
WT SPG11
S1955A-SPG11

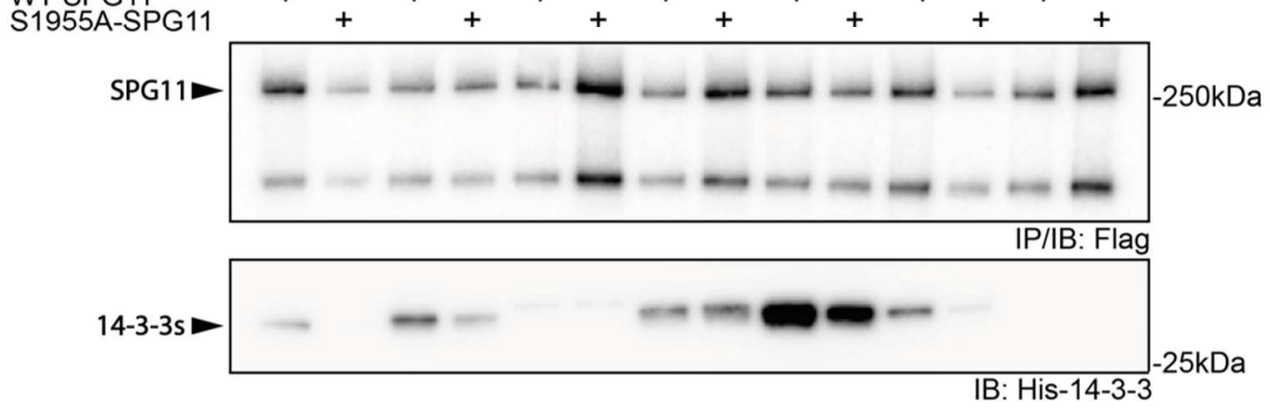

Figure 3 - Phospho-Ser1955 is key for 14-3-3 binding to SPG11. (A) Schematic of the putative 14-3-3 binding sites predicted by 14-3-3-Pred. Ser1955, highlighted in light blue, was also reported to be a phosphorylated residue by PhosphoSitePlus ${ }^{\circledR}$, and confirmed by phospho-peptide enrichment experiments performed in this work. (B) Binding affinity of 14-3-3s for phosphorylated SPG11-Ser1955 was confirmed through generation of phospho-deficient S1955A-mutant by site-directed mutagenesis. The nonphosphorylatable mutant displays $\sim 50 \%$ reduction in binding as compared to the WT counterpart. 
A.

\begin{tabular}{|l|l|}
\hline Putative kinase & Score \\
\hline PKA & 0.799 \\
\hline RSK & 0.607 \\
\hline CaM-II & 0.483 \\
\hline GSK3 & 0.481 \\
\hline PKG & 0.435 \\
\hline DNAPK & 0.371 \\
\hline cdc2 & 0.366 \\
\hline CKI & 0.365 \\
\hline p38MAPK & 0.320 \\
\hline ATM & 0.267 \\
\hline CKII & 0.246 \\
\hline cdk5 & 0.181 \\
\hline PKB & 0.167 \\
\hline PKC & 0.116 \\
\hline
\end{tabular}

B.
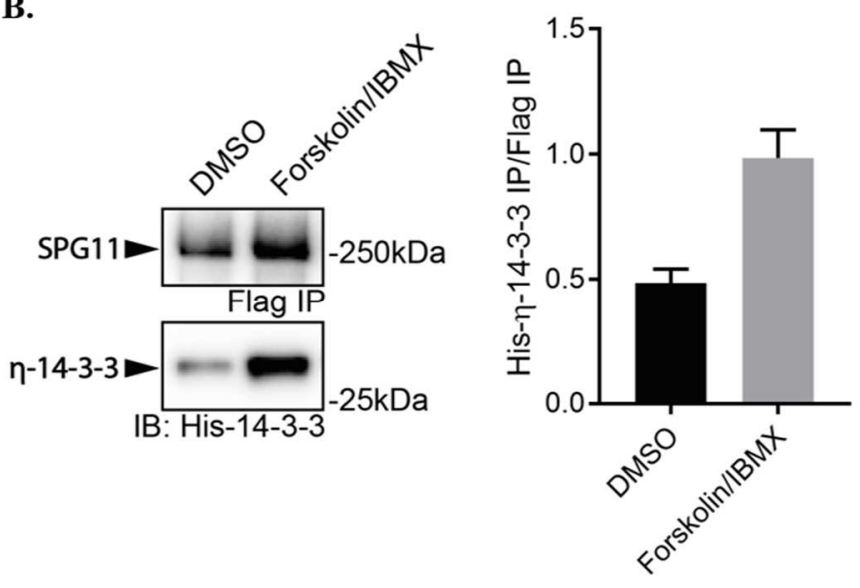

PKA consensus R-X-(X)-S/T-X

SPG11 sequence R-V-H-S-T
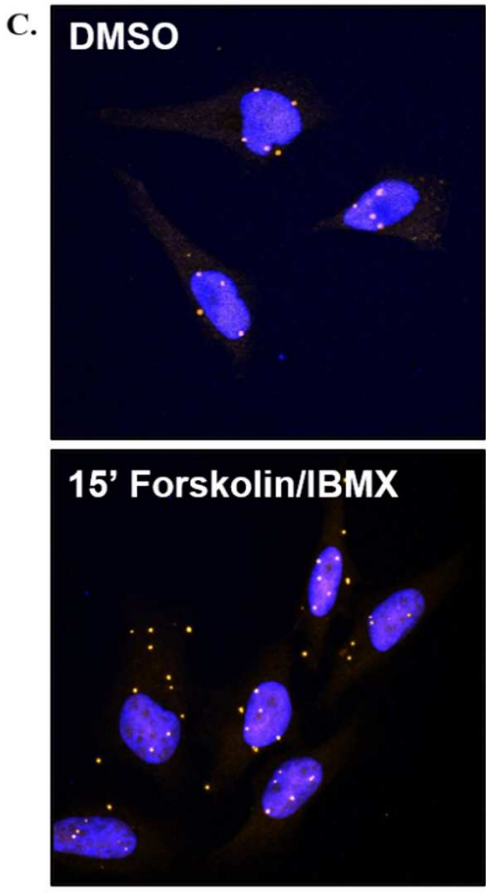
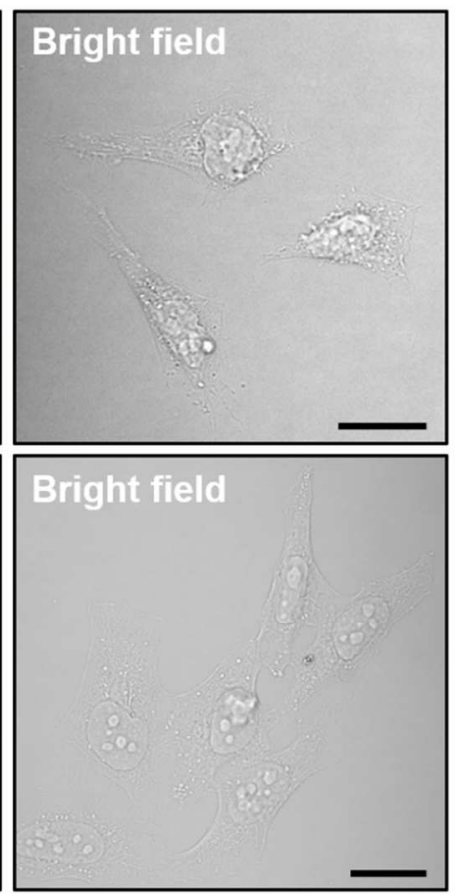
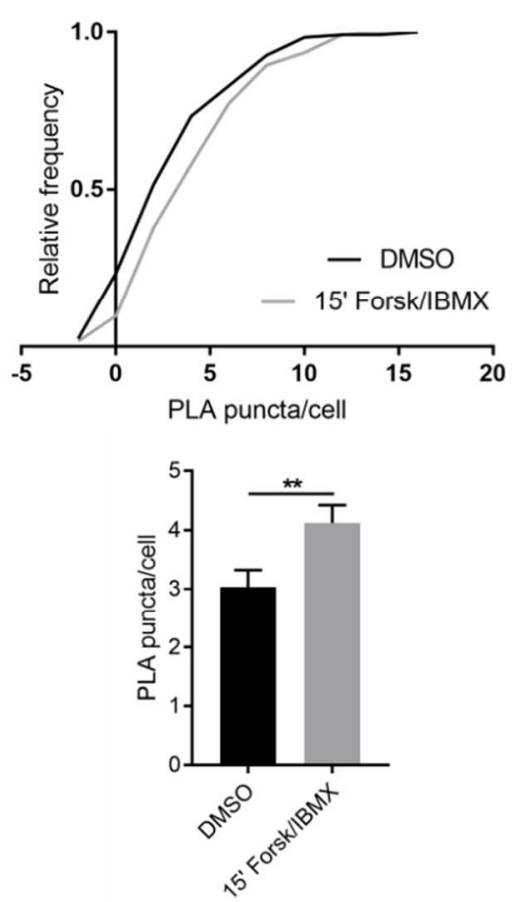

Figure 4 - Ser 1955 on SPG11 is phosphorylated by PKA. (A) Summary of the putative kinases phosphorylating SPG11 on Ser1955, with relative confidence score, as obtained from NetPhos 3.1. The PKA consensus sequence is also indicated and aligned against the SPG11 motif containing Ser1955. (B) Endogenous SPG11 was pulled down from genome-edited monoclonal lines taking advantage of the 3xFlag tag sequence inserted at the endogenous locus. 15 minutes treatment with Forskolin/IBMX increases binding affinity for $\eta-14-3-3$, suggesting that phosphorylation by PKA on Ser1955 is the signal regulating interaction $(n=2)$. (C) PLA experiments confirmed (i) interaction between SPG11 and 14-3-3s at the endogenous level and (ii) increased binding in the presence of PKA activation. (PLA reactions were performed between rabbit $\alpha$-Flag and mouse $\alpha$-pan-14-3-3 antibodies. Average background signal detected in naïve cells, employed as a negative control for the reaction, was subtracted to all measurements; $n=2, \sim 125$ cells counted/condition). 
A.

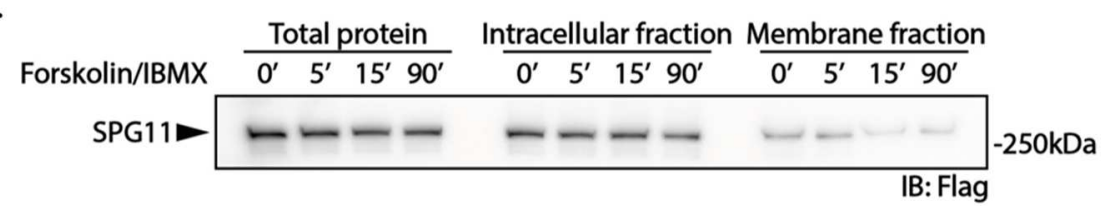

B.

15'

DMSO
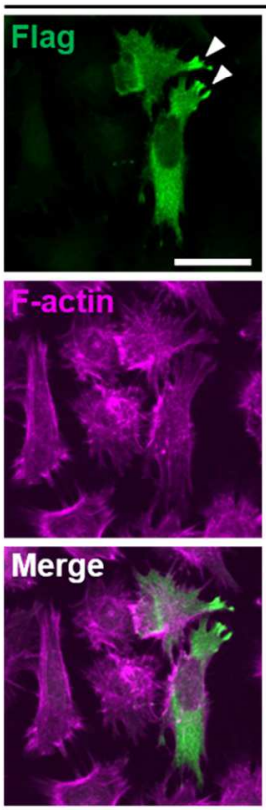

WT
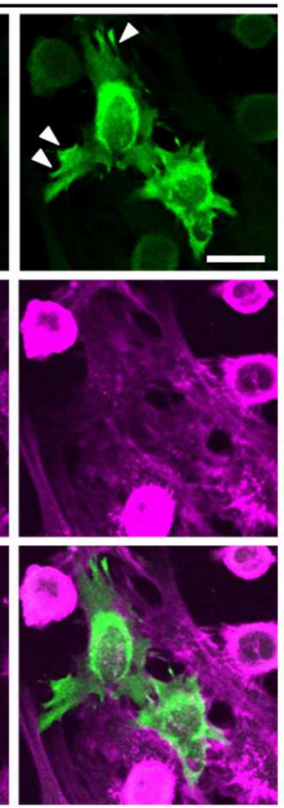

SA
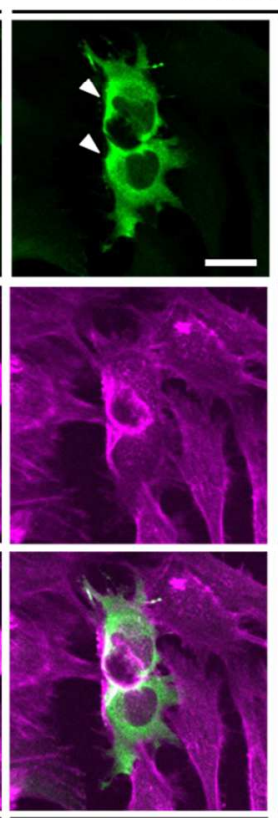

WT
IIBMX

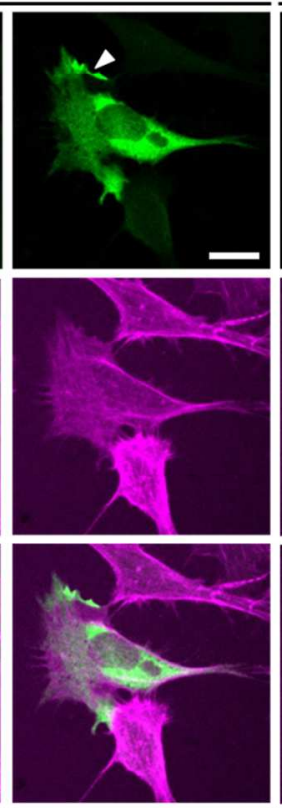

SA
90'

Forsk/IBMX

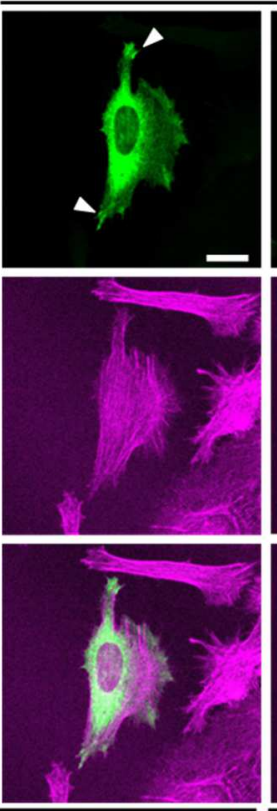

WT

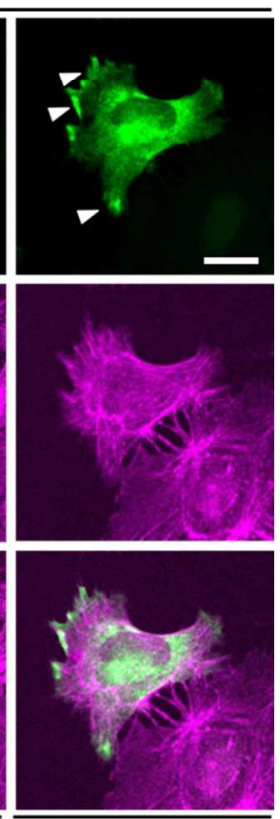

SA

Figure 5 - PKA-mediated phosphorylation initiates SPG11 traffic from the plasma membrane. (A) Cell surface biotinylation assay showing endogenous SPG11 internalisation upon 15 minutes treatment with Forskolin, suggesting that PKA activation is the stimulus to initiate SPG11 trafficking towards intracellular compartments. (B) Representative images of HeLa cells transfected with WT or S1955A-SPG11 and treated with Forskolin/IBMX for 15 and 90 minutes show that only the WT protein is able to respond to PKA activation, suggesting that the mechanism is mediated by 14-3-3 binding. White arrowheads indicate SPG11 enrichment at protrusion-like structures, which is reduced after 15 minutes treatment with Forskolin/IBMX. Scale bar $=20 \mu \mathrm{m}$. 


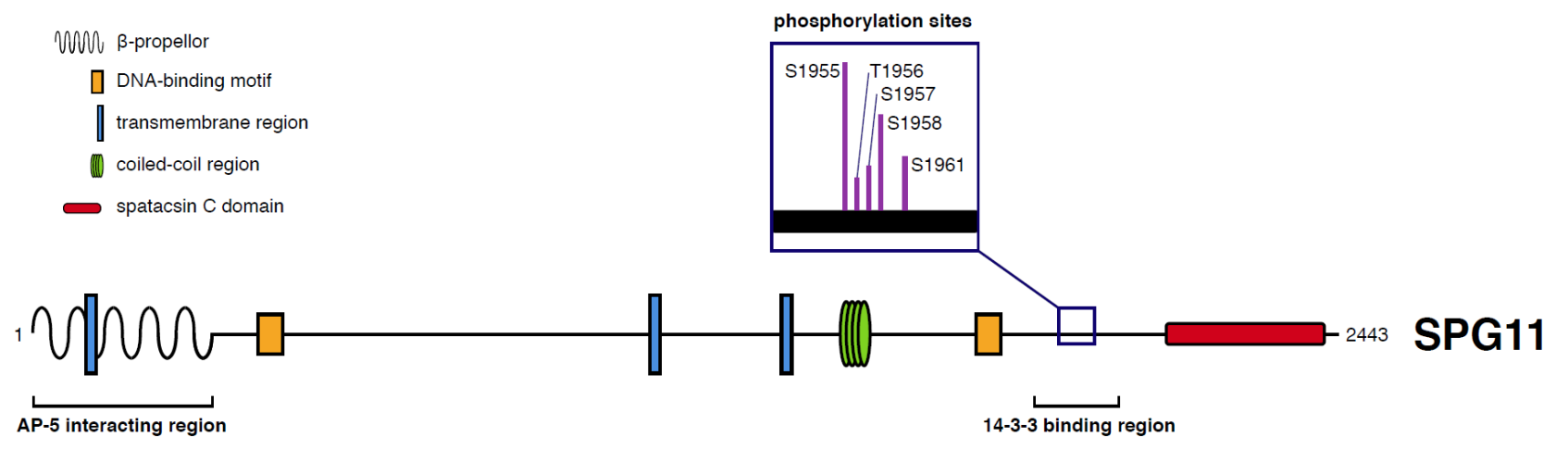

Figure 6 - Cartoon representation of SPG11 domain organisation. Canonical motifs for interactions with binding partners and transmembrane domains that have been predicted or identified by other groups (Hirst et al., 2013; Stevanin et al., 2007) and confirmed by in silico data from this study are summarised. The AP5interacting region (Hirst et al., 2013) and the spatacsin $\mathrm{C}$ domain, which shares sequence and structure similarity with the Vps16 protein, identified in the study by Patto and O'Kane (Patto \& O'kane, 2020) are also included, together with the phospho-peptide responsible for the binding of 14-3-3s identified in this study. 


\section{Bibliography}

Belluzzi, E., Gonnelli, A., Cirnaru, M. D., Marte, A., Plotegher, N., Russo, I., Civiero, L., Cogo, S., Carrion, M. P., Franchin, C., Arrigoni, G., Beltramini, M., Bubacco, L., Onofri, F., Piccoli, G., \& Greggio, E. (2016). LRRK2 phosphorylates pre-synaptic N-ethylmaleimide sensitive fusion (NSF) protein enhancing its ATPase activity and SNARE complex disassembling rate. Molecular Neurodegeneration, 11(1), 1. https://doi.org/10.1186/s13024-015-0066-Z

Blackstone, C. (2018). Converging cellular themes for the hereditary spastic paraplegias. In Current Opinion in Neurobiology (Vol. 51, pp. 139-146). Elsevier Ltd. https://doi.org/10.1016/j.conb.2018.04.025

Blackstone, C. (2020). Early-onset hereditary spastic paraplegia: the possibility of a genetic diagnosis. Developmental Medicine \& Child Neurology, 62(9), 1011-1011. https://doi.org/10.1111/dmcn.14564

Blom, N., Gammeltoft, S., \& Brunak, S. (1999). Sequence and structure-based prediction of eukaryotic protein phosphorylation sites. Journal of Molecular Biology, 294(5), 1351-1362. https://doi.org/10.1006/jmbi.1999.3310

Blom, N., Sicheritz-Pontén, T., Gupta, R., Gammeltoft, S., \& Brunak, S. (2004). Prediction of posttranslational glycosylation and phosphorylation of proteins from the amino acid sequence. In Proteomics (Vol. 4, Issue 6, pp. 1633-1649). John Wiley \& Sons, Ltd. https://doi.org/10.1002/pmic.200300771

Boutry, M., Branchu, J., Lustremant, C., Pujol, C., Pernelle, J., Matusiak, R., Seyer, A., Poirel, M., Chu-Van, E., Pierga, A., Dobrenis, K., Puech, J. P., Caillaud, C., Durr, A., Brice, A., Colsch, B., Mochel, F., El Hachimi, K. H., Stevanin, G., \& Darios, F. (2018). Inhibition of Lysosome Membrane Recycling Causes Accumulation of Gangliosides that Contribute to Neurodegeneration. Cell Reports, 23(13), 3813-3826. https://doi.org/10.1016/j.celrep.2018.05.098

Boutry, M., Pierga, A., Matusiak, R., Branchu, J., Houllegatte, M., Ibrahim, Y., Balse, E., El Hachimi, K. H., Brice, A., Stevanin, G., \& Darios, F. (2019). Loss of spatacsin impairs cholesterol trafficking and calcium homeostasis. Communications Biology, 2(1). https://doi.org/10.1038/s42003-019-0615-z

Branchu, J., Boutry, M., Sourd, L., Depp, M., Leone, C., Corriger, A., Vallucci, M., Esteves, T., Matusiak, R., Dumont, M., Muriel, M. P., Santorelli, F. M., Brice, A., El Hachimi, K. H., 
Stevanin, G., \& Darios, F. (2017). Loss of spatacsin function alters lysosomal lipid clearance leading to upper and lower motor neuron degeneration. Neurobiology of Disease, 102, 21-37. https://doi.org/10.1016/j.nbd.2017.02.007

Buchan, D. W. A., \& Jones, D. T. (2019). The PSIPRED Protein Analysis Workbench: 20 years on. Nucleic Acids Research, 47(W1), W402-W407. https://doi.org/10.1093/nar/gkz297

Chang, J., Lee, S., \& Blackstone, C. (2014). Spastic paraplegia proteins spastizin and spatacsin mediate autophagic lysosome reformation. Journal of Clinical Investigation, 124(12), 52495262. https://doi.org/10.1172/JCI77598

Civiero, L., Cogo, S., Kiekens, A., Morganti, C., Tessari, I., Lobbestael, E., Baekelandt, V., Taymans, J.-M., Chartier-Harlin, M.-C., Franchin, C., Arrigoni, G., Lewis, P. A., Piccoli, G., Bubacco, L., Cookson, M. R., Pinton, P., \& Greggio, E. (2017). PAK6 Phosphorylates 14-3-3 $\gamma$ to Regulate Steady State Phosphorylation of LRRK2. Frontiers in Molecular Neuroscience, 10, 417. https://doi.org/10.3389/fnmol.2017.00417

Cornell, B., \& Toyo-oka, K. (2017). 14-3-3 proteins in brain development: Neurogenesis, neuronal migration and neuromorphogenesis. In Frontiers in Molecular Neuroscience (Vol. 10, p. 318). Frontiers Media S.A. https://doi.org/10.3389/fnmol.2017.00318

Dalvai, M., Loehr, J., Jacquet, K., Huard, C. C., Roques, C., Herst, P., Côté, J., \& Doyon, Y. (2015). A Scalable Genome-Editing-Based Approach for Mapping Multiprotein Complexes in Human Cells. Cell Reports, 13(3), 621-633. https://doi.org/10.1016/j.celrep.2015.09.009

Darios, F., Mochel, F., \& Stevanin, G. (2020). Lipids in the Physiopathology of Hereditary Spastic Paraplegias. In Frontiers in Neuroscience (Vol. 14, p. 74). Frontiers Media S.A. https://doi.org/10.3389/fnins.2020.00074

Doench, J. G., Fusi, N., Sullender, M., Hegde, M., Vaimberg, E. W., Donovan, K. F., Smith, I., Tothova, Z., Wilen, C., Orchard, R., Virgin, H. W., Listgarten, J., \& Root, D. E. (2016). Optimized sgRNA design to maximize activity and minimize off-target effects of CRISPR-Cas9. Nature Biotechnology, 34(2), 184-191. https://doi.org/10.1038/nbt.3437

Folts, C. J., Scott-Hewitt, N., Pröschel, C., Mayer-Pröschel, M., \& Noble, M. (2016). Lysosomal Reacidification Prevents Lysosphingolipid-Induced Lysosomal Impairment and Cellular Toxicity. PLoS Biology, 14(12), e1002583. https://doi.org/10.1371/journal.pbio.1002583

Foote, M., \& Zhou, Y. (2012). 14-3-3 proteins in neurological disorders. International Journal of Biochemistry and Molecular Biology, 3(2), 152-164. www.ijbmb.org 
Gervasi, N., Hepp, R., Tricoire, L., Zhang, J., Lambolez, B., Paupardin-Tritsch, D., \& Vincent, P. (2007). Dynamics of protein kinase A signaling at the membrane, in the cytosol, and in the nucleus of neurons in mouse brain slices. Journal of Neuroscience, 27(11), 2744-2750. https://doi.org/10.1523/JNEUROSCI.5352-06.2007

Hamaguchi, T., Nakamuta, S., Funahashi, Y., Takano, T., Nishioka, T., Shohag, M. H., Yura, Y., Kaibuchi, K., \& Amano, M. (2015). In vivo Screening for Substrates of Protein Kinase A Using a Combination of Proteomic Approaches and Pharmacological Modulation of Kinase Activity. Cell Structure and Function, 40(1), 1-12. https://doi.org/10.1247/csf.14014

Hirst, J., Borner, G. H. H., Edgar, J., Hein, M. Y., Mann, M., Buchholz, F., Antrobus, R., \& Robinson, M. S. (2013). Interaction between AP-5 and the hereditary spastic paraplegia proteins SPG11 and SPG15. Molecular Biology of the Cell, 24(16), 2558-2569. https://doi.org/10.1091/mbc.E13-03-0170

Hsu, P. D., Scott, D. A., Weinstein, J. A., Ran, F. A., Konermann, S., Agarwala, V., Li, Y., Fine, E. J., Wu, X., Shalem, O., Cradick, T. J., Marraffini, L. A., Bao, G., \& Zhang, F. (2013). DNA targeting specificity of RNA-guided Cas9 nucleases. Nature Biotechnology, 31(9), 827-832. https://doi.org/10.1038/nbt.2647

Hu, Y., Flockhart, I., Vinayagam, A., Bergwitz, C., Berger, B., Perrimon, N., \& Mohr, S. E. (2011). An integrative approach to ortholog prediction for disease-focused and other functional studies. BMC Bioinformatics, 12(1), 357. https://doi.org/10.1186/1471-2105-12-357

Huttlin, E., Bruckner, R., Navarrete-Perea, J., Cannon, J., Baltier, K., Gebreab, F., Gygi, M., Thornock, A., Zarraga, G., Tam, S., Szpyt, J., Panov, A., Parzen, H., Fu, S., Golbazi, A., Maenpaa, E., Stricker, K., Thakurta, S. G., Rad, R., ... Gygi, S. (2020). Dual Proteome-scale Networks Reveal Cell-specific Remodeling of the Human Interactome. BioRxiv, 2020.01.19.905109. https://doi.org/10.1101/2020.01.19.905109

Kaplan, A., Ottmann, C., \& Fournier, A. E. (2017). 14-3-3 adaptor protein-protein interactions as therapeutic targets for CNS diseases. In Pharmacological Research (Vol. 125, pp. 114-121). Academic Press. https://doi.org/10.1016/j.phrs.2017.09.007

Khundadze, M., Kollmann, K., Koch, N., Biskup, C., Nietzsche, S., Zimmer, G., Hennings, J. C., Huebner, A. K., Symmank, J., Jahic, A., Ilina, E. I., Karle, K., Schöls, L., Kessels, M., Braulke, T., Qualmann, B., Kurth, I., Beetz, C., \& Hübner, C. A. (2013). A Hereditary Spastic Paraplegia Mouse Model Supports a Role of ZFYVE26/SPASTIZIN for the Endolysosomal System. PLoS 
Genetics, 9(12), e1003988. https://doi.org/10.1371/journal.pgen.1003988

Khundadze, M., Ribaudo, F., Hussain, A., Rosentreter, J., Nietzsche, S., Thelen, M., Winter, D., Hoffmann, B., Afzal, M. A., Hermann, T., de Heus, C., Piskor, E. M., Kosan, C., Franzka, P., von Kleist, L., Stauber, T., Klumperman, J., Damme, M., Proikas-Cezanne, T., \& Hübner, C. A. (2019). A mouse model for SPG48 reveals a block of autophagic flux upon disruption of adaptor protein complex five. Neurobiology of Disease, 127, 419-431. https://doi.org/10.1016/j.nbd.2019.03.026

Klebe, S., Stevanin, G., \& Depienne, C. (2015). Clinical and genetic heterogeneity in hereditary spastic paraplegias: From SPG1 to SPG72 and still counting. Revue Neurologique, 171(7). https://doi.org/10.1016/j.neurol.2015.02.017ï

Kumar, M., Gouw, M., Michael, S., Sámano-Sánchez, H., Pancsa, R., Glavina, J., Diakogianni, A., Valverde, J. A., Bukirova, D., Signalyševa, J., Palopoli, N., Davey, N. E., Chemes, L. B., \& Gibson, T. J. (2020). ELM-the eukaryotic linear motif resource in 2020. Nucleic Acids Research, 48(D1), D296-D306. https://doi.org/10.1093/nar/gkz1030

Lo Giudice, T., Lombardi, F., Santorelli, F. M., Kawarai, T., \& Orlacchio, A. (2014). Hereditary spastic paraplegia: Clinical-genetic characteristics and evolving molecular mechanisms. In Experimental Neurology (Vol. 261, pp. 518-539). Academic Press Inc. https://doi.org/10.1016/j.expneurol.2014.06.011

Madeira, F., Tinti, M., Murugesan, G., Berrett, E., Stafford, M., Toth, R., Cole, C., MacKintosh, C., \& Barton, G. J. (2015). 14-3-3-Pred: Improved methods to predict 14-3-3-binding phosphopeptides. Bioinformatics, 31(14), 2276-2283. https://doi.org/10.1093/bioinformatics/btv133

McFerrin, M. B., Chi, X., Cutter, G., \& Yacoubian, T. A. (2017). Dysregulation of 14-3-3 proteins in neurodegenerative diseases with Lewy body or Alzheimer pathology. Annals of Clinical and Translational Neurology, 4(7), 466-477. https://doi.org/10.1002/acn3.421

Mellacheruvu, D., Wright, Z., Couzens, A. L., Lambert, J. P., St-Denis, N. A., Li, T., Miteva, Y. V., Hauri, S., Sardiu, M. E., Low, T. Y., Halim, V. A., Bagshaw, R. D., Hubner, N. C., Al-Hakim, A., Bouchard, A., Faubert, D., Fermin, D., Dunham, W. H., Goudreault, M., ... Nesvizhskii, A. I. (2013). The CRAPome: A contaminant repository for affinity purification-mass spectrometry data. Nature Methods, 10(8), 730-736. https://doi.org/10.1038/nmeth.2557

Murmu, R. P., Martin, E., Rastetter, A., Esteves, T., Muriel, M. P., El Hachimi, K. H., Denora, P. S., 
Dauphin, A., Fernandez, J. C., Duyckaerts, C., Brice, A., Darios, F., \& Stevanin, G. (2011). Cellular distribution and subcellular localization of spatacsin and spastizin, two proteins involved in hereditary spastic paraplegia. Molecular and Cellular Neuroscience, 47(3), 191202. https://doi.org/10.1016/j.mcn.2011.04.004

Namkoong, S., Kim, C. K., Cho, Y. L., Kim, J. H., Lee, H., Ha, K. S., Choe, J., Kim, P. H., Won, M. H., Kwon, Y. G., Shim, E. B., \& Kim, Y. M. (2009). Forskolin increases angiogenesis through the coordinated cross-talk of PKA-dependent VEGF expression and Epac-mediated PI3K/Akt/eNOS signaling. Cellular Signalling, 21(6), 906-915. https://doi.org/10.1016/j.cellsig.2009.01.038

Patra, C., \& Brady, M. F. (2018). Biochemistry, cAMP. In StatPearls. StatPearls Publishing. http://www.ncbi.nlm.nih.gov/pubmed/30571052

Patto, A. L., \& O'kane, C. J. (2020). Distant homologies and domain conservation of the Hereditary Spastic Paraplegia protein SPG11/ALS5/spatacsin. BioRxiv, 2020.03.08.982389. https://doi.org/10.1101/2020.03.08.982389

Pensato, V., Castellotti, B., Gellera, C., Pareyson, D., Ciano, C., Nanetti, L., Salsano, E., Piscosquito, G., Sarto, E., Eoli, M., Moroni, I., Soliveri, P., Lamperti, E., Chiapparini, L., Di Bella, D., Taroni, F., \& Mariotti, C. (2014). Overlapping phenotypes in complex spastic paraplegias SPG11, SPG15, SPG35 and SPG48. Brain, 137(7), 1907-1920. https://doi.org/10.1093/brain/awu121

Pérez-Brangulí, F., Mishra, H. K., Prots, I., Havlicek, S., Kohl, Z., Saul, D., Rummel, C., DorcaArevalo, J., Regensburger, M., Graef, D., Sock, E., Blasi, J., Groemer, T. W., SchlötzerSchrehardt, U., Winkler, J., \& Winner, B. (2014). Dysfunction of spatacsin leads to axonal pathology in SPG11-linked hereditary spastic paraplegia. Human Molecular Genetics, 23(18), 4859-4874. https://doi.org/10.1093/hmg/ddu200

Pozner, T., Schray, A., Regensburger, M., Lie, D. C., Schlötzer-Schrehardt, U., Winkler, J., Turan, S., \& Winner, B. (2018). Tideglusib Rescues Neurite Pathology of SPG11 iPSC Derived Cortical Neurons. Frontiers in Neuroscience, 12, 914. https://doi.org/10.3389/fnins.2018.00914

Puntervoll, P., Linding, R., Gemünd, C., Chabanis-Davidson, S., Mattingsdal, M., Cameron, S., Martin, D. M. A., Ausiello, G., Brannetti, B., Costantini, A., Ferrè, F., Maselli, V., Via, A., Cesareni, G., Diella, F., Superti-Furga, G., Wyrwicz, L., Ramu, C., McGuigan, C., ... Gibson, T. J. (2003). ELM server: A new resource for investigating short functional sites in modular 
eukaryotic proteins. Nucleic Acids Research, 31(13), 3625-3630. https://doi.org/10.1093/nar/gkg545

Renvoisé, B., Chang, J., Singh, R., Yonekawa, S., FitzGibbon, E. J., Mankodi, A., Vanderver, A., Schindler, A. B., Toro, C., Gahl, W. A., Mahuran, D. J., Blackstone, C., \& Pierson, T. M. (2014). Lysosomal abnormalities in hereditary spastic paraplegia types SPG15 and SPG11. Annals of Clinical and Translational Neurology, 1(6), 379-389. https://doi.org/10.1002/acn3.64

Sassone-Corsi, P. (2012). The Cyclic AMP pathway. Cold Spring Harbor Perspectives in Biology, 4(12), a011148. https://doi.org/10.1101/cshperspect.a011148

Sharma, A., Toepfer, C. N., Ward, T., Wasson, L., Agarwal, R., Conner, D. A., Hu, J. H., \& Seidman, C. E. (2018). CRISPR/Cas9-Mediated Fluorescent Tagging of Endogenous Proteins in Human Pluripotent Stem Cells. Current Protocols in Human Genetics, 96(1), 21.11.1-21.11.20. https://doi.org/10.1002/cphg.52

Sluchanko, N. N., \& Gusev, N. B. (2012). Oligomeric structure of 14-3-3 protein: What do we know $\begin{array}{llll}\text { about monomers? } & \text { FEBS } & \text { Letters, }\end{array}$ https://doi.org/10.1016/j.febslet.2012.10.048

Sluchanko, N. N., \& Gusev, N. B. (2017). Moonlighting chaperone-like activity of the universal regulatory 14-3-3 proteins. The FEBS Journal, 284(9), 1279-1295. https://doi.org/10.1111/febs.13986

Stevanin, G., Azzedine, H., Denora, P., Boukhris, A., Tazir, M., Lossos, A., Rosa, A. L., Lerer, I., Hamri, A., Alegria, P., Loureiro, J., Tada, M., Hannequin, D., Anheim, M., Goizet, C., GonzalezMartinez, V., Le Ber, I., Forlani, S., Iwabuchi, K., ... Durr, A. (2008). Mutations in SPG11 are frequent in autosomal recessive spastic paraplegia with thin corpus callosum, cognitive decline and lower motor neuron degeneration. Brain, 131(3), 772-784. https://doi.org/10.1093/brain/awm293

Stevanin, G., Santorelli, F. M., Azzedine, H., Coutinho, P., Chomilier, J., Denora, P. S., Martin, E., Ouvrard-Hernandez, A.-M., Tessa, A., Bouslam, N. N., Lossos, A., Charles, P., Loureiro, J. L., Elleuch, N., Confavreux, C., Cruz, V. T., Ruberg, M., Leguern, E., Grid, D., ... Brice, A. (2007). Mutations in SPG11, encoding spatacsin, are a major cause of spastic paraplegia with thin corpus callosum. https://doi.org/10.1038/ng1980

Taus, T., Köcher, T., Pichler, P., Paschke, C., Schmidt, A., Henrich, C., \& Mechtler, K. (2011). Universal and confident phosphorylation site localization using phosphoRS. Journal of 
Proteome Research, 10(12), 5354-5362. https://doi.org/10.1021/pr200611n

Tomkins, J. E., Ferrari, R., Vavouraki, N., Hardy, J., Hardy, J., Hardy, J., Hardy, J., Hardy, J., Lovering, R. C., Lewis, P. A., Lewis, P. A., Lewis, P. A., McGuffin, L. J., Manzoni, C., \& Manzoni, C. (2020). PINOT: An intuitive resource for integrating protein-protein interactions. Cell Communication and Signaling, 18(1), 92. https://doi.org/10.1186/s12964-020-00554-5

Vantaggiato, C., Panzeri, E., Castelli, M., Citterio, A., Arnoldi, A., Santorelli, F. M., Liguori, R., Scarlato, M., Musumeci, O., Toscano, A., Clementi, E., \& Bassi, M. T. (2019). ZFYVE26/SPASTIZIN and SPG11/SPATACSIN mutations in hereditary spastic paraplegia types AR-SPG15 and AR-SPG11 have different effects on autophagy and endocytosis. Autophagy, 15(1), 34-57. https://doi.org/10.1080/15548627.2018.1507438

Varga, R.-E., Khundadze, M., Damme, M., Nietzsche, S., Hoffmann, B., Stauber, T., Koch, N., Hennings, J. C., Franzka, P., Huebner, A. K., Kessels, M. M., Biskup, C., Jentsch, T. J., Qualmann, B., Braulke, T., Kurth, I., Beetz, C., \& Hübner, C. A. (2015). In Vivo Evidence for Lysosome Depletion and Impaired Autophagic Clearance in Hereditary Spastic Paraplegia Type SPG11. PLOS Genetics, 11(8), e1005454. https://doi.org/10.1371/journal.pgen.1005454 